\title{
Association between brain-derived neurotrophic factor gene polymorphisms and fibromyalgia in a Korean population: a multicenter study
}

Dong-Jin Park', Seong-Ho Kim², Seong-Su Nah³ ${ }^{3}$ Ji Hyun Lee ${ }^{4}$, Seong-Kyu Kim, Yeon-Ah Lee ${ }^{6}$, Seung-Jae Hong ${ }^{6}$, Hyun-Sook Kim ${ }^{7}$, Hye-Soon Lee ${ }^{8}$, Hyoun Ah Kim ${ }^{9}$, Chung-II Joung ${ }^{10}$, Sang-Hyon Kim ${ }^{11}$ and Shin-Seok Lee ${ }^{1 *}$ (D)

\begin{abstract}
Background: Several lines of evidence imply that brain-derived neurotrophic factor (BDNF) is involved in the pathophysiology of fibromyalgia (FM); in this regard, patients with FM have altered blood and cerebrospinal fluid levels of BDNF. In this study, we explored the association between BDNF gene polymorphisms and FM susceptibility and the severity of symptoms.

Methods: In total, 409 patients with FM and 423 healthy controls in 10 medical centers were enrolled from the Korean nationwide FM survey. The alleles and genotypes at 10 positions in the BDNF gene were genotyped.

Results: The allele and genotype frequencies of BDNF rs1 1030104 differed significantly between the patients with FM and the controls $(P=0.031)$. The $G G$ genotype of rs 11030104 had a protective effect against FM $(P=0.016)$, and the $\mathrm{G}$ allele of rs 11030104 was negatively associated with the presence of FM compared with the $\mathrm{A}$ allele $(P=0$. 013). In comparison, although the allele and genotype frequencies of BDNF rs12273539 did not differ between the two groups, the $\Pi$ genotype of BDNF rs 12273539 was associated with susceptibility to FM $(P=0.038)$. Haplotype analyses implied that some BDNF haplotypes have a protective effect against FM. Finally, several genotypes and haplotypes of the BDNF gene contributed to specific symptoms of FM.

Conclusions: This study is the first to evaluate the associations between BDNF gene polymorphisms and FM. Our results imply that some BDNF single-nucleotide polymorphisms and haplotypes are associated with susceptibility to, and contribute to the symptoms of, FM.
\end{abstract}

Keywords: Fibromyalgia, Brain-derived neurotrophic factor, Genetics, Polymorphism

\section{Background}

Fibromyalgia (FM) is a common rheumatic syndrome characterized by chronic widespread pain, and is often accompanied by diverse symptoms including fatigue, sleep disorders, memory loss, joint stiffness, and affective distress [1]. The prevalence of FM in the general population is reportedly $1-5 \%$, and it is more prevalent among women than men [2]. Although its pathogenesis is

\footnotetext{
* Correspondence: shinseok@chonnam.ac.kr

${ }^{1}$ Division of Rheumatology, Department of Internal Medicine, Chonnam National University Medical School and Hospital, 42 Jebong-ro, Dong-gu, Gwangju 61469, Republic of Korea

Full list of author information is available at the end of the article
}

unclear, FM is recognized as an outcome of the interactions of multiple genetic, psychological, neurobiological, and environmental factors [3].

The familial aggregation observed among patients with FM implies that genetic factors are important contributors to the etiology of FM [4]. Recent genetic studies have advanced our understanding of the pathogenesis of FM. These studies have shown that certain gene polymorphisms alter pain sensitivity and increase susceptibility to FM [5]. In particular, polymorphisms of genes involved in the pain transmission pathway, such as the serotoninergic, dopaminergic, and catecholaminergic systems, have received much attention as possible genetic factors in FM 
$[6,7]$. However, those genetic factors do not fully account for the pathophysiology and symptoms of FM. Therefore, efforts to identify other genetic factors that contribute to FM are ongoing.

Brain-derived neurotrophic factor (BDNF) is involved in neuronal survival, growth, and differentiation during development of the central and peripheral nervous systems [8]. BDNF is important in the transmission of physiologic or pathologic pain [9]. BDNF is responsible for modulation of nociceptive inputs and enhanced hyperalgesia by a $\mathrm{N}$-methyl-D-aspartate (NMDA) receptor-mediated mechanism [10]. Moreover, dysregulation of BDNF in the dorsal root ganglion (DRG) and spinal cord contributes to chronic pain hypersensitivity [11]. In addition, several lines of evidence have converged to imply that BDNF is involved in the pathophysiology of FM. Indeed, patients with FM have been shown to have altered serum and plasma levels of BDNF compared to healthy controls [12-14].

However, whether polymorphisms of the BDNF gene are associated with FM remains an open question. The objective of this study was to evaluate the associations between $B D N F$ gene polymorphisms and FM susceptibility and clinical symptoms, using a large population of ethnically homogenous Koreans.

\section{Methods}

\section{Study design and population}

We performed a multicenter, nationwide FM cohort study (the Korean Nationwide FM Survey) in the Korean population. In the Korean Nationwide FM Survey, we established a prospective cohort to evaluate the pathophysiology of FM, and the clinical manifestations and outcomes of Korean patients with FM. The study participants were recruited from the outpatient rheumatology clinics of 10 medical centers. In this study, a cross-sectional design was employed to evaluate the association between $B D N F$ gene polymorphisms and susceptibility to, and symptom severity of, FM. As reported previously [15], we enrolled 409 patients with FM (382 women and 27 men) with a mean (SD) age of 48.1 (10.9) years. At the time of the initial diagnosis, patients with FM were diagnosed according to the classification criteria for FM proposed by the American College of Rheumatology (ACR) in 1990 [1]. The mean (SD) symptom duration before diagnosis was 8.5 (8.3) years, and the mean (SD) disease duration after initial diagnosis was 1.9 (3.0) years. Based on health surveys for chronic pain, we recruited 423 healthy controls (397 women, 25 men) with a mean (SD) age of 45.5 (12.5) years and no history of chronic pain, including FM. Healthy controls were recruited randomly, without matching for age or sex, among the individuals visiting the general health examination clinics at each medical center. This research complied with the Helsinki Declaration, and written informed consent was obtained from all participants at the time of recruitment. Exactly the same informed consult form (ICF) and study protocol were provided to the independent Institutional Review Board/Ethics Committee (IRB/EC) at each medical center, and each IRB/EC reviewed the appropriateness of the protocol and risks and benefits to the study participants. Ultimately, the IRB/EC at each medical center independently approved this study without revision of the ICF or study protocol.

\section{Procedures}

The patients with FM were interviewed at the time of enrollment to determine their demographics and clinical characteristics, including age, sex, body mass index, and symptom and disease duration. In addition, at enrollment, peripheral venous blood was sampled and then stored in an ethylenediaminetetraacetate (EDTA)-coated tube. Tender points were assessed by thumb palpation according to the standardized tender point survey protocol [16]. The number of tender points was assessed at 18 sites on the body. The intensity at each tender point was assessed by determining the tender point score as follows: 0 , no tenderness; 1, light tenderness (confirming answer when asked); 2 , moderate tenderness (spontaneous verbal response); and 3 , severe tenderness (moving away). Therefore, the number of tender points ranged from 0 to 18 , and the possible total scores of the tender points ranged from 0 to 54 . Furthermore, extensive clinical assessments of patients with FM enrolled in the cohort were undertaken using a self-report questionnaire and semi-structured questionnaires. The Korean version of the Fibromyalgia Impact Questionnaire (FIQ) was used to assess the functional abilities and severity of FM [17], and the Brief Fatigue Inventory (BFI) and the Beck Depression Inventory (BDI) were used to evaluate the severity of fatigue and depression, respectively $[18,19]$. The 36-item Medical Outcomes Study Short-Form Health Survey (SF-36) was used to access the quality of life of the patients with FM [20]. In addition, we also evaluated the severity of anxiety using the State-Trait Anxiety Inventory (STAI)-I and STAI-II [21].

The patients had been treated with standard medications for FM, based on the clinical judgment of their attending rheumatologist. Concomitant medications, used at the time of enrollment, included tricyclic antidepressants (TCA), selective serotonin reuptake inhibitors (SSRI), serotonin-norepinephrine reuptake inhibitors (SNRI), pregabalin, gabapentin, nonsteroidal anti-inflammatory drugs (NSAIDs), acetaminophen, benzodiazepine, tramadol, and muscle relaxants.

\section{Genotyping of BDNF polymorphisms}

The assay reagents for rs2883187(C > T), rs7103873 (G > C), rs7103411 $(\mathrm{C}>\mathrm{T}), \mathrm{rs} 10835210(\mathrm{C}>\mathrm{A}), \operatorname{rs} 11030104(\mathrm{~A}>$ G), rs12273539(C > T), rs11030102(C > G), rs11030101(A > $\mathrm{T}), \operatorname{rs} 6265(\mathrm{G}>\mathrm{A})$ and $\operatorname{rs} 7124442(\mathrm{C}>\mathrm{T})$ in the $B D N F$ gene 
were designed by Applied Biosystems (Applied Biosystems). The reagents consisted of TaqMan MGB probes (FAM and VIC dye-labeled). Each reaction $(10 \mu \mathrm{L})$ comprised $0.125 \mu \mathrm{L}$ of $40 \mathrm{X}$ reagents, $5 \mu \mathrm{L}$ of $2 \mathrm{X}$ TaqMan Genotyping Master Mix (Applied Biosystems) and $2 \mu \mathrm{L}$ of $50 \mathrm{ng}$ genomic DNA. The PCR conditions were 1 cycle at $95^{\circ} \mathrm{C}$ for $10 \mathrm{~min}$, followed by 40 cycles at $95{ }^{\circ} \mathrm{C}$ for $15 \mathrm{~s}$ and $60{ }^{\circ} \mathrm{C}$ for $1 \mathrm{~min}$. The PCR reactions were performed using an ABI plus instrument (Applied Biosystems). The samples were read and analyzed using ABI plus software (Applied Biosystems). The sequences of the primers used for TaqMan probe genotyping of the $B D N F$ gene are summarized in Table 1.

\section{Statistical analysis}

Statistical analyses were performed using IBM SPSS statistics (SPSS version 21; IBM SPSS Inc., Chicago, IL, USA). $P$ values $<0.05$ were considered to indicate statistical significance. Each $B D N F$ gene polymorphism was tested for Hardy-Weinberg equilibrium. The genotype and haplotype frequencies of the $B D N F$ single-nucleotide polymorphisms (SNPs) were compared between the patients with FM and controls by Fisher's exact test or Pearson's chi-squared test. The association between each BDNF genotype and haplotype and susceptibility to FM was defined by logistic regression analysis. Analysis of covariance

Table 1 Primer sequences used for TaqMan probe genotyping of BDNF

\begin{tabular}{|c|c|c|}
\hline Regions & Primers & Primer sequence $\left(5^{\prime} \rightarrow 3^{\prime}\right)$ \\
\hline \multirow[t]{2}{*}{ rs 2883187} & Forward & GTGAGGCATCCGGCCCGGCTGGGGA \\
\hline & Reverse & CGGAGCGCGGTCTCGGCAGCTCCCC \\
\hline \multirow[t]{2}{*}{ rs 7103873} & Forward & AGGACCTTITACCCCCAAATGTAGA \\
\hline & Reverse & ACTAAATGAAAAACCATTCTITAAA \\
\hline \multirow[t]{2}{*}{ rs 7103411} & Forward & GGAGCGCACTGTAAAGATACTGATA \\
\hline & Reverse & GAACACGAATGTGAGATCAATGTTG \\
\hline \multirow[t]{2}{*}{ rs 10835210} & Forward & CTTAACTGTAAAGCACAGGAAAGTG \\
\hline & Reverse & TCATTACTTGTAGCTTAATGCAGGA \\
\hline \multirow[t]{2}{*}{ rs 11030104} & Forward & ATTAAAAAGCAGATAACACTACCAC \\
\hline & Reverse & TACTAACTGTCCTACAATTTCCTGT \\
\hline \multirow[t]{2}{*}{ rs 12273539} & Forward & ACTCAATGCTTCATCACTTCTGCTC \\
\hline & Reverse & GATCAGGACAGAGTCCTTGGAGTGC \\
\hline \multirow[t]{2}{*}{ rs 11030102} & Forward & CTACTTCTCAGTTCTGAGGCATGGA \\
\hline & Reverse & TTACAAAAAGACACATACATGCAAT \\
\hline \multirow[t]{2}{*}{ rs 11030101} & Forward & GATACTCTATTATAGCAAAGAAGAA \\
\hline & Reverse & GATAATTCATTGAGCCATCCTGTT \\
\hline \multirow[t]{2}{*}{ rs 6265} & Forward & TCCTCATCCAACAGCTCTTCTATCA \\
\hline & Reverse & GTGTTCGAAAGTGTCAGCCAATGAT \\
\hline \multirow[t]{2}{*}{ rs 7124442} & Forward & AAGGAAGCTGCATAAAGTTGACATA \\
\hline & Reverse & AGCAGATATTCCAAGCATTCCTTAC \\
\hline
\end{tabular}

$B D N F$ brain-derived neurotrophic factor
(ANCOVA), adjusted for age and sex, was used to explore the differences in the clinical measurements of the patients with FM according to $B D N F$ genotype and haplotype. Haplotype structures were constructed and their frequencies estimated by combined allele analysis using PHASE v2.1.1 software (Department of Statistics, University of Washington, Seattle, WA, USA). We carried out a permutation test for the null hypothesis that the patients with FM and the healthy controls are random draws from a common set of haplotype frequencies (number of permutations performed $=10,000$ ).

\section{Results}

BDNF genotypes and alleles and their association with clinical measurements

The BDNF SNPs were successfully genotyped in all enrolled subjects, except for 5 controls with $B D N F$ rs 2883187 , 1 patient and 16 controls with $B D N F$ rs7103873, 2 controls with $B D N F$ rs7103411, 1 patient and 10 controls with $B D N F$ rs10835210, 2 patients and 3 controls with $B D N F$ rs11030104, 1 control with $B D N F$ rs12273539, 1 patient and 1 control with $B D N F$ rs11030102, 1 patient and 3 controls with $B D N F$ rs11030101, 1 patient and 4 controls with $B D N F$ rs6265, and 2 patients and 2 controls with $B D N F$ rs7124442. The genotype distributions of the BDNF SNPs were consistent with Hardy-Weinberg equilibrium in both the patients and controls.

Among the BDNF SNPs, the allele and genotype frequencies of BDNF SNP rs11030104 were significantly different between the patients with FM and controls. Furthermore, patients with the GG genotype of rs11030104 were found less frequently in patients with FM after adjusting for age and sex (OR 0.619; 95\% confidence interval (CI) 0.419-0.0913; $P=0.016$ ). In addition, the $G$ allele was negatively associated with the presence of FM compared to the A allele $(\mathrm{OR}=0.781,95 \% \mathrm{CI}$ $0.641-0.950, P=0.013)$. In comparison, although the allele and genotype frequencies of the SNPs of $B D N F$ rs12273539 were not significantly different between the patients with FM and controls, the TT genotype of rs12273539 was found more frequently in patients with FM in the age-adjusted and sex-adjusted model (OR 2.586; 95\% CI 1.052-6.360; $P=0.038$ ) (Table 2).

Within the FM cohort, patients with the CG genotype of $B D N F$ rs11030102 had more severe fatigue symptoms (measured by the BFI) and anxiety symptoms (measured by the STAI-I) than did the other genotypes $(P=0.001$ and $P=0.032$, respectively). Furthermore, both rs11030101 and rs10835210 were associated with the trait of anxiety (measured by the STAI-II) in patients with FM $(P=0.029$ and $P=0.033$, respectively). No associations were observed between clinical measurements and the other BDNF SNPs (Table 3). 
Table 2 Genotype and allele analyses of BDNF in patients with fibromyalgia and healthy controls ${ }^{\mathrm{a}}$

\begin{tabular}{|c|c|c|c|c|c|c|}
\hline Marker & Genotype/allele & Contol, $n(\%)$ & Fibromyalgia, $n(\%)$ & Exact $p$ value ${ }^{b}$ & OR $(95 \% \mathrm{Cl}), p$ value $^{c}$ & $\begin{array}{l}\text { OR }(95 \% \text { Cl), } p \text { value, } \\
\text { adjusted by age, sex }\end{array}$ \\
\hline \multirow[t]{5}{*}{ rs2883187 } & $\mathrm{C} / \mathrm{C}$ & $115(27.5)$ & $100(24.4)$ & 0.218 & 1 & 1 \\
\hline & $\mathrm{C} / \mathrm{T}$ & $220(52.6)$ & $208(50.9)$ & & $1.087(0.783-1.510), p=0.617$ & $1.044(0.747-1.458), p=0.802$ \\
\hline & $\mathrm{T} / \mathrm{T}$ & $83(19.9)$ & $101(24.7)$ & & $1.399(0.943-2.078), p=0.096$ & $1.340(0.897-2.002), p=0.152$ \\
\hline & C & $450(53.8)$ & $408(49.9)$ & 0.119 & 1 & \\
\hline & $\mathrm{T}$ & $386(46.2)$ & $410(50.1)$ & & $1.172(0.966-1.421), p=0.108$ & $1.147(0.943-1.395), p=0.171$ \\
\hline \multirow[t]{5}{*}{ rs7103873 } & $\mathrm{G} / \mathrm{G}$ & $113(27.8)$ & $98(24.0)$ & 0.245 & 1 & 1 \\
\hline & $C / G$ & $210(51.6)$ & $208(51.0)$ & & $1.142(0.820-1.591), p=0.432$ & $1.110(0.791-1.556), p=0.546$ \\
\hline & $\mathrm{C} / \mathrm{C}$ & $84(20.6)$ & $102(25.0)$ & & $1.400(0.943-2.080), p=0.095$ & $1.345(0.899-2.010), p=0.149$ \\
\hline & G & $436(53.6)$ & $404(49.5)$ & 0.112 & 1 & \\
\hline & C & $378(46.4)$ & $412(50.5)$ & & $1.176(0.968-1.429), p=0.102$ & $1.153(0.946-1.405), p=0.158$ \\
\hline \multirow[t]{5}{*}{ rs7103411 } & $\mathrm{C} / \mathrm{C}$ & $120(28.5)$ & $128(31.3)$ & 0.638 & 1 & 1 \\
\hline & $\mathrm{C} / \mathrm{T}$ & $208(49.4)$ & $198(48.4)$ & & $0.892(0.651-1.224), p=0.48$ & $0.884(0.641-1.220), p=0.454$ \\
\hline & $\mathrm{T} / \mathrm{T}$ & $93(22.1)$ & $83(20.3)$ & & $0.837(0.568-1.232), p=0.366$ & $0.865(0.584-1.280), p=0.468$ \\
\hline & C & $448(53.2)$ & $454(55.5)$ & 0.374 & 1 & \\
\hline & $\mathrm{T}$ & $394(46.8)$ & $364(44.5)$ & & $0.912(0.751-1.106), p=0.348$ & $0.925(0.76-1.125), p=0.435$ \\
\hline \multirow[t]{5}{*}{ rs10835210 } & $\mathrm{C} / \mathrm{C}$ & $204(49.4)$ & $196(48.0)$ & 0.725 & 1 & 1 \\
\hline & $\mathrm{A} / \mathrm{C}$ & $175(42.4)$ & $172(42.2)$ & & $1.023(0.767-1.364), p=0.877$ & $0.991(0.740-1.326), p=0.949$ \\
\hline & $\mathrm{A} / \mathrm{A}$ & $34(8.2)$ & $40(9.8)$ & & $1.224(0.745-2.014), p=0.425$ & $1.183(0.714-1.957), p=0.514$ \\
\hline & C & $583(70.6)$ & $564(69.1)$ & 0.554 & 1 & \\
\hline & A & $243(29.4)$ & $252(30.9)$ & & $1.072(0.868-1.324), p=0.518$ & $1.048(0.846-1.297), p=0.671$ \\
\hline \multirow[t]{5}{*}{ rs11030104 } & A/A & $101(24.0)$ & $126(31.0)$ & 0.031 & 1 & 1 \\
\hline & $A / G$ & $205(48.8)$ & $196(48.2)$ & & $0.766(0.553-1.063), p=0.111$ & $0.758(0.544-1.057), p=0.102$ \\
\hline & $\mathrm{G} / \mathrm{G}$ & $114(27.1)$ & $85(20.9)$ & & $0.598(0.407-0.877), p=0.009$ & $0.619(0.419-0.913), p=0.016$ \\
\hline & A & $407(48.5)$ & $448(55.0)$ & 0.009 & 1 & \\
\hline & G & $433(51.5)$ & $366(45.0)$ & & $0.768(0.633-0.932), p=0.007$ & $0.781(0.641-0.95), p=0.013$ \\
\hline \multirow[t]{5}{*}{ rs12273539 } & $\mathrm{C} / \mathrm{C}$ & $283(67.1)$ & $268(65.4)$ & 0.101 & 1 & 1 \\
\hline & $C / T$ & $132(31.3)$ & $125(30.5)$ & & $1(0.744-1.345), p=1$ & $1.009(0.747-1.362), p=0.955$ \\
\hline & $\mathrm{T} / \mathrm{T}$ & $7(1.7)$ & $17(4.1)$ & & $2.564(1.047-6.282), p=0.039$ & $2.586(1.052-6.36), p=0.038$ \\
\hline & C & $698(82.7)$ & $661(80.6)$ & 0.299 & 1 & \\
\hline & $\mathrm{T}$ & $146(17.3)$ & $159(19.4)$ & & $1.150(0.897-1.475), p=0.27$ & $1.161(0.902-1.493), p=0.246$ \\
\hline \multirow[t]{4}{*}{ rs11030102 } & $\mathrm{C} / \mathrm{C}$ & 419 (99.3) & $402(98.5)$ & 0.334 & 1 & 1 \\
\hline & $C / G$ & $3(0.7)$ & $6(1.5)$ & & $2.085(0.518-8.392), p=0.301$ & $2.129(0.524-8.649), p=0.291$ \\
\hline & C & 841 (99.6) & 810 (99.3) & 0.335 & 1 & \\
\hline & G & $3(0.4)$ & $6(0.7)$ & & $2.077(0.518-8.326), p=0.302$ & $2.12(0.524-8.58), p=0.292$ \\
\hline \multirow[t]{5}{*}{ rs11030101 } & $\mathrm{A} / \mathrm{A}$ & $208(49.5)$ & $197(48.3)$ & 0.752 & 1 & 1 \\
\hline & $\mathrm{A} / \mathrm{T}$ & $178(42.4)$ & $172(42.2)$ & & $1.020(0.766-1.358), p=0.891$ & $0.985(0.737-1.317), p=0.92$ \\
\hline & $\mathrm{T} / \mathrm{T}$ & $34(8.1)$ & $39(9.6)$ & & $1.211(0.735-1.996), p=0.452$ & $1.152(0.694-1.912), p=0.583$ \\
\hline & A & $594(70.7)$ & $566(69.4)$ & 0.585 & 1 & \\
\hline & $\mathrm{T}$ & $246(29.3)$ & $250(30.6)$ & & $1.067(0.864-1.316), p=0.548$ & $1.036(0.837-1.283), p=0.743$ \\
\hline \multirow[t]{4}{*}{ rs6265 } & $\mathrm{G} / \mathrm{G}$ & $96(22.9)$ & $87(21.3)$ & 0.770 & 1 & 1 \\
\hline & $A / G$ & $204(48.7)$ & $197(48.3)$ & & $1.066(0.751-1.512), p=0.722$ & $1.017(0.712-1.451), p=0.928$ \\
\hline & A/A & $119(28.4)$ & $124(30.4)$ & & $1.150(0.783-1.688), p=0.476$ & $1.110(0.752-1.638), p=0.599$ \\
\hline & G & $396(47.3)$ & $371(45.5)$ & 0.496 & 1 & \\
\hline
\end{tabular}


Table 2 Genotype and allele analyses of BDNF in patients with fibromyalgia and healthy controls ${ }^{\mathrm{a}}$ (Continued)

\begin{tabular}{|c|c|c|c|c|c|c|}
\hline Marker & Genotype/allele & Contol, $n(\%)$ & Fibromyalgia, n (\%) & Exact $p$ value ${ }^{b}$ & OR $(95 \% \mathrm{Cl}), p$ value & $\begin{array}{l}\text { OR }(95 \% \mathrm{Cl}), p \text { value, } \\
\text { adjusted by age, sex }\end{array}$ \\
\hline & A & $442(52.7)$ & $445(54.5)$ & & $1.075(0.886-1.304), p=0.466$ & $1.058(0.869-1.287), p=0.575$ \\
\hline \multirow[t]{5}{*}{ rs7124442 } & $\mathrm{C} / \mathrm{C}$ & $2(0.5)$ & $0(0)$ & 0.574 & 1 & 1 \\
\hline & $\mathrm{C} / \mathrm{T}$ & $51(12.1)$ & $47(11.5)$ & & $718,117.521(0-\operatorname{lnf}), p=0.972$ & $683,123.831(0-\operatorname{Inf}), p=0.972$ \\
\hline & $\mathrm{T} / \mathrm{T}$ & $368(87.4)$ & $360(88.5)$ & & $762,294.038(0-\ln f), p=0.971$ & $682,163.974(0-\operatorname{Inf}), p=0.972$ \\
\hline & C & $55(6.5)$ & $47(5.8)$ & 0.590 & 1 & \\
\hline & $\mathrm{T}$ & $787(93.5)$ & $767(94.2)$ & & $1.140(0.763-1.705), p=0.521$ & $1.078(0.716-1.623), p=0.72$ \\
\hline
\end{tabular}

BDNF brain-derived neurotrophic factor

${ }^{a}$ Missing data were excluded from the analyses: BDNF rs2883187 (5 controls), BDNF rs7103873 (1 patient and 16 controls), BDNF rs7103411 (2 controls), BDNF rs10835210 (1 patient and 10 controls), BDNF rs 11030104 (2 patients and 3 controls), BDNF rs 12273539 (1 control), BDNF rs 11030102 ( 1 patient and 1 control), $B D N F$ rs 11030101 (1 patient and 3 controls), BDNF rs6265 (4 controls and 1 patient), and BDNF rs7124442 ( 2 patients and 2 controls)

${ }^{b}$ Value was determined by Fisher's exact test or $x^{2}$ test

'Logistic regression analyses were used to calculate the OR $(95 \% \mathrm{Cl}$; confidence interval)

\section{Haplotype frequencies and clinical measurements}

Among the 39 haplotype structures included in the haplotype analysis of BDNF SNPs, seven frequent haplotypes (TGACCGCTGC, TATCCAACCT, TGACCACTGC, TAACTACCCT, TATCCGACCT, TAACTGCCCT, and CAACCACCGC) had a frequency of $>1 \%$ in the patients and controls. Although not shown in Table 4, the total frequency of the other haplotype structures was 30 (3.8\%) for patients and 46 (6\%) for controls. These haplotypes showed significantly different distributions between the patients with FM and the controls ( $P=0.0001$; Table 4).

Among the frequent haplotypes, the TGACCACTGC haplotype was found less frequently in the patients with FM after adjusting for age and sex (OR 0.004, 95\% CI 0.0-0.026, $P<0.001$; Table 5). Interestingly, the TATC CGACCT and TAACTGCCCT haplotypes were not detected in patients with FM (Table 5) (both $P>0.05$ ). In the clinical measures, only anxiety, assessed using the STAI-II score, was significantly different among the patients according to BDNF haplotype (Table 6).

\section{Discussion}

To our knowledge, we were the first to investigate the association between BDNF SNPs and FM. We found that the allele and genotype frequencies of $B D N F$ rs11030104 were significantly different between the patients with FM and the controls. In comparison, although the allele and genotype frequencies of $B D N F$ rs12273539 were not significantly different between the patients with FM and the controls, the TT genotype of $B D N F$ rs12273539 was associated with susceptibility to FM. In addition to the individual SNPs, certain BDNF haplotypes may be protective against FM or contribute to its symptoms. Therefore, our data imply that BDNF gene polymorphisms contribute to the development and symptom severity of FM in the Korean population.

Neurotrophic factors are a family of closely related proteins involved in neuronal survival, growth, and differentiation during development of the nervous system
[9]. Neurotrophins comprise four structurally related factors: BDNF, nerve growth factor (NGF), neurotrophin 3 (NT-3), and neurotrophin 4/5 (NT-4/5). Neurotrophins play important roles in the transmission of physiologic and pathologic pain [22]. In particular, BDNF plays key roles in chronic pain conditions. BDNF is synthesized in the DRG, and is transported to the central terminals of the primary afferents in the spinal dorsal horn, where it is involved in the modulation of painful stimuli [9]. BDNF contributes to central sensitization by modulating nociceptive inputs and enhancing hyperalgesia through NMDA-receptor-mediated responses [23]. For these reasons, researchers have been interested in the role of BDNF in chronic pain disorders, including FM [24]. In addition, BDNF plays a role in depressive disorder, which is frequently comorbid with FM; indeed, the serum level of BDNF is altered in patients with depression $[25,26]$. Moreover, it can be normalized by antidepressants such as milnacipran [26], which are frequently used in the treatment of FM.

Several clinical studies have evaluated the role of BDNF in the pathogenesis of FM. Patients with FM have increased levels of BDNF in blood [12, 14] and cerebrospinal fluid [27] compared to healthy controls, implying that BDNF is involved in the pathophysiology of FM. In particular, Zanette et al. reported that serum BDNF levels are inversely associated with the pressure-pain threshold in patients with FM [13]. Furthermore, increased serum BDNF mediates the disinhibition of motor cortex excitability and the function of the descending inhibitory pain modulation system in patients with FM [28]. In fact, recent studies have shown that disruptions in default mode network (DMN) connectivity may be associated with impaired pain modulation, leading to the chronic pain seen in FM $[29,30]$. Furthermore, certain $B D N F$ polymorphisms have an effect on specific aspects of brain function such as DMN connectivity, which is currently considered to be central in the pathogenesis of FM [31]. These findings could be a potential explanation that 


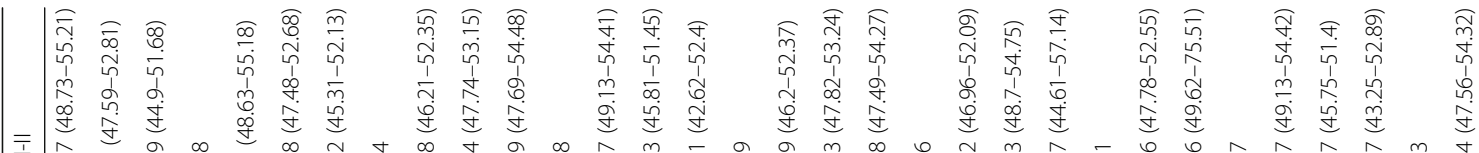

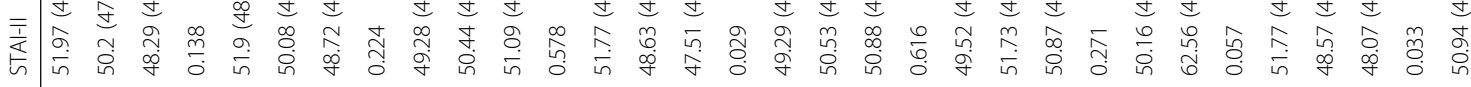

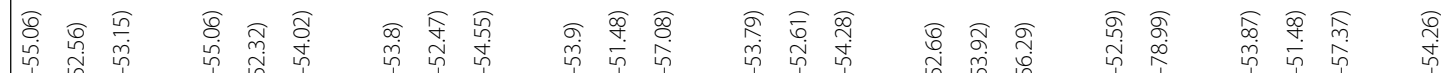

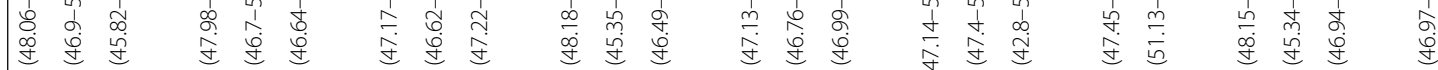

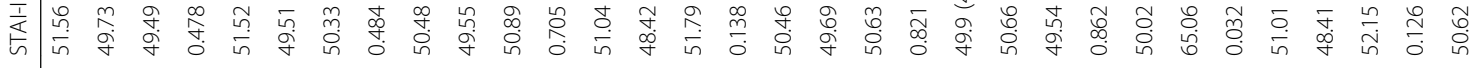

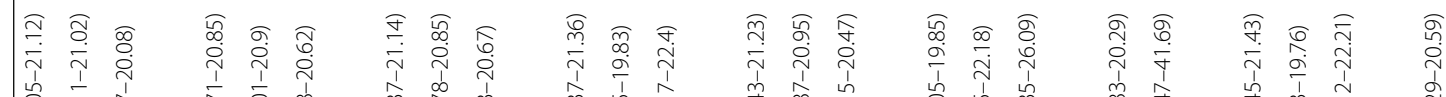

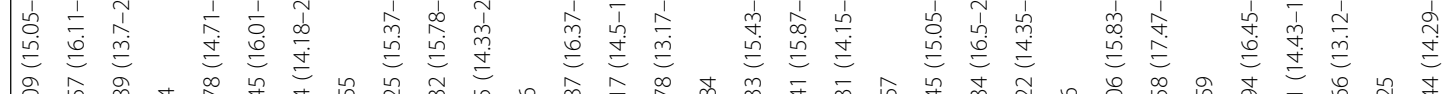

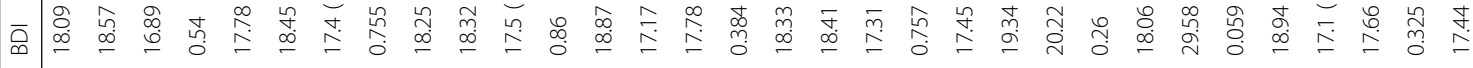

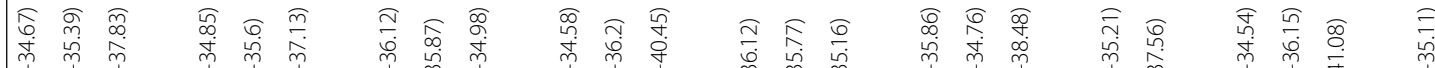

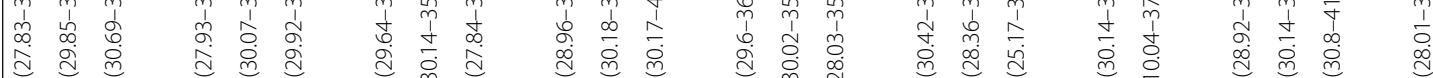

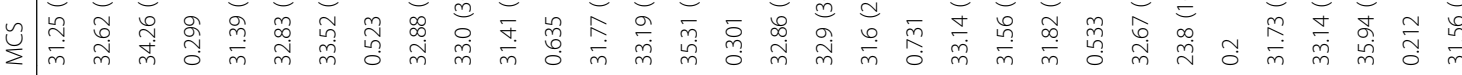

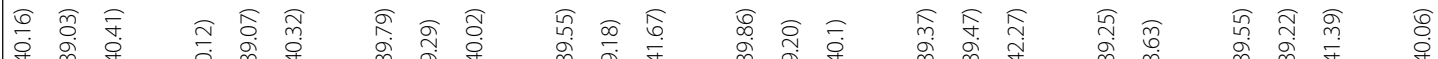

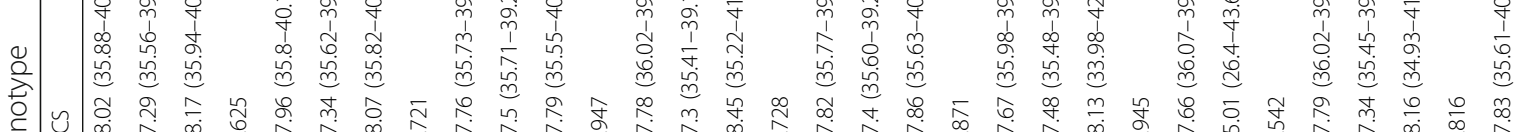

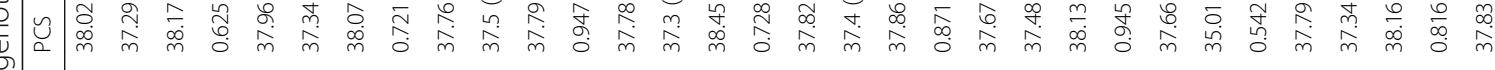

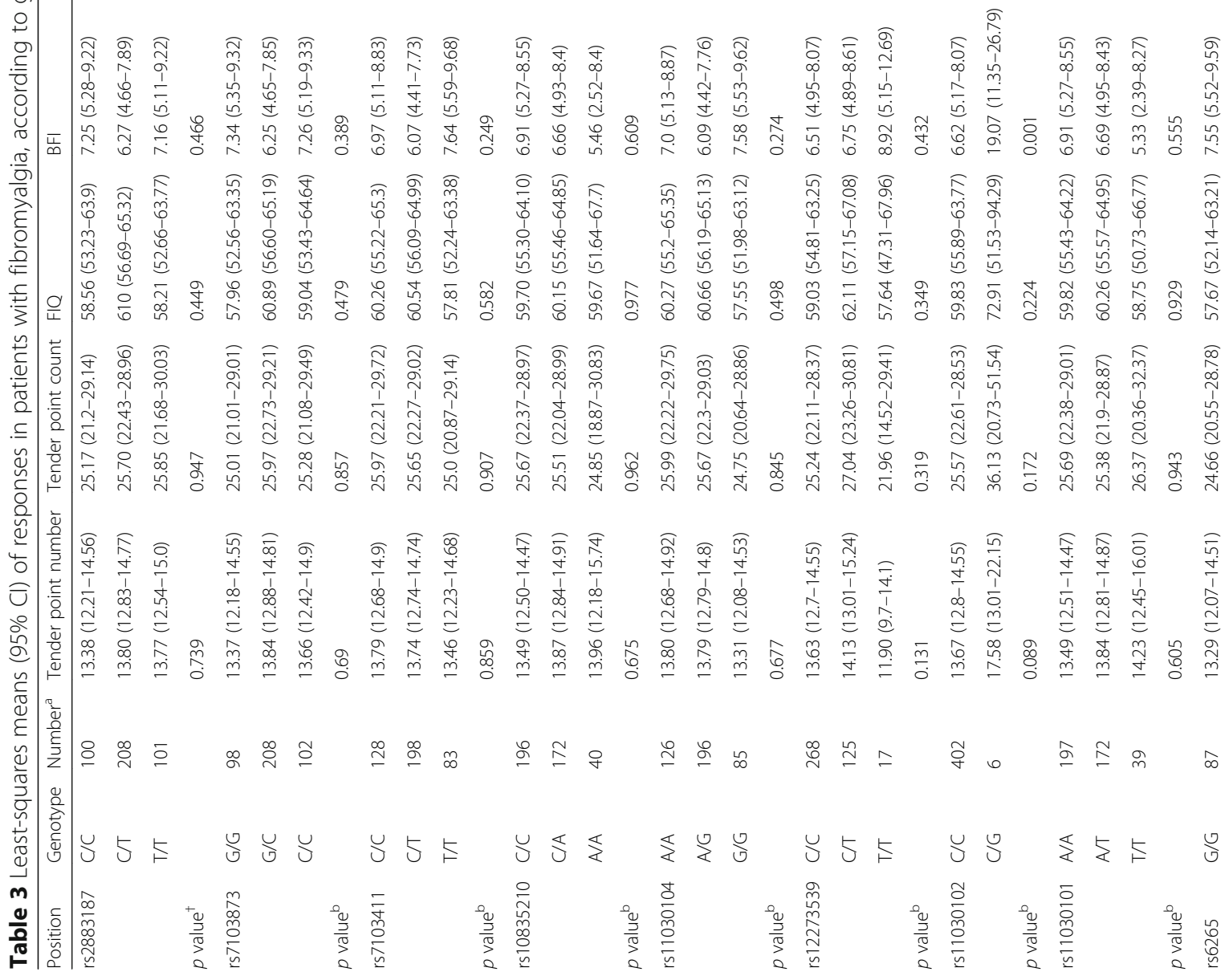




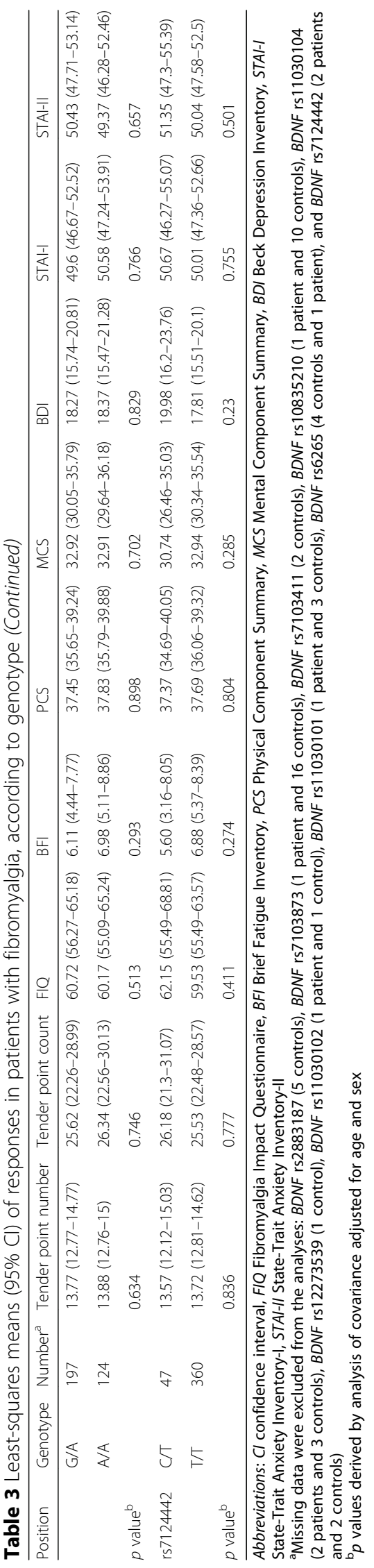


Table 4 Estimates of haplotype frequencies in patients with fibromyalgia $(n=393)$ and healthy controls $(n=388)^{2}$

\begin{tabular}{lllll}
\hline Combined alleles $^{a}$ & All subjects & Controls & Fibromyalgia & $p$ value $^{b}$ \\
\hline TGACCGCTGC & $29.6 \pm 0.75$ & $20.22 \pm 0.9$ & $38.87 \pm 0.88$ & 0.0001 \\
TATCCAACCT & $20.16 \pm 0.44$ & $12.94 \pm 0.57$ & $27.29 \pm 0.52$ & \\
TGACCACTGC & $14.8 \pm 0.75$ & $25.26 \pm 0.89$ & $4.47 \pm 0.88$ & \\
TAACTACCCT & $11.97 \pm 0.42$ & $6.99 \pm 0.5$ & $16.89 \pm 0.53$ & \\
TATCCGACCT & $8.94 \pm 0.45$ & $15.76 \pm 0.59$ & $2.21 \pm 0.52$ & \\
TAACTGCCCT & $5.74 \pm 0.42$ & $9.8 \pm 0.5$ & $1.72 \pm 0.53$ & \\
CAACCACCGC & $3.20 \pm 0.17$ & $2.06 \pm 0.22$ & $4.33 \pm 0.24$ &
\end{tabular}

${ }^{a}$ Data are percentages \pm SE

${ }^{a}$ Missing data were excluded $(n=51)$. Among 39 haplotype structures, 7 haplotypes with frequency of at least $1 \%$ in both the patients and controls are presented

${ }^{b} p$ values for permutation test of the null hypothesis that cases and controls are random draws from a common set of haplotype frequencies (number of permutations $=10,000$ )

supports the existence of a mechanistic link between $B D N F$ polymorphisms and FM. However, although multiple lines of evidence imply a role for BDNF in the pathogenesis of FM, $B D N F$ polymorphisms in these patients have not been investigated extensively.

In this study, we found that certain BDNF SNPs are associated with susceptibility to FM. The GG genotype and the $\mathrm{G}$ allele of $B D N F$ rs11030104 exert a protective effect against FM. In contrast, although the allele and genotype frequencies of $B D N F$ rs12273539 did not differ between the patients with FM and controls, the TT genotype of $B D N F$ rs12273539 was associated with susceptibility to FM. To date, only one study has evaluated associations between $B D N F$ gene polymorphisms and FM. Xiao et al. [32] evaluated whether the BDNF Val66Met polymorphism was associated with FM; their results implied that the $B D N F$ Val66 Met SNP is associated with a subgroup of patients with FM with high-sensitivity $\mathrm{C}$-reactive protein and high body mass index. Nevertheless, the relative distribution of the BDNF Val66Met SNP did not differ between the patients with FM and healthy controls. Similarly, in our study, BDNF Val66Val Met was not associated with susceptibility to FM. However, our data demonstrate that other BDNF SNPs, such as rs11030104 and rs12273539, were associated with the risk of FM in a Korean population.

Furthermore, our data imply that certain BDNF haplotypes exert a protective effect against FM. A haplotype refers to a particular set of closely linked alleles that are inherited as a unit, and haplotype analysis can reveal the pattern of genetic variation associated with certain diseases [33]. Several haplotypes of certain genes are reportedly significantly associated with FM. Diatchenko et al. [34] reported that the ACCG haplotype, which consists of four SNPs (rs6269, rs4633, rs4818, and rs4680) of the catechol-O-methyltransferase (COMT) gene, is associated with both FM susceptibility and symptom severity $[35,36]$. Similarly, we also suggested that a particular haplotype of $T R P V 2$ may be associated with susceptibility to FM [37]. In the current study, our findings imply that BDNF haplotypes may be involved in the pathophysiology of FM.

Notably, we failed to uncover a direct association between $B D N F$ gene polymorphisms and pain-related symptom scales such as the tender point number and count. However, those polymorphisms were related to certain psychological symptoms in patients with FM. In particular, certain BDNF SNPs and haplotypes were associated with anxiety symptoms. Since patients with FM have a significantly higher prevalence of anxiety disorders (13-63.8\%) [38], our findings imply that $B D N F$ gene polymorphisms may indirectly affect FM through their effect on anxiety. However, diverse factors affect the development of FM, including psychological symptoms such as anxiety, so our results should be interpreted carefully.

This study had several limitations. First, it was of a case-control design. Because the purpose of this study was to evaluate the role of BDNF SNPs associated with susceptibility to FM, we adopted a target-gene-based approach. Therefore, like the majority of SNP studies, we selected candidate SNPs for a case-control analysis of their association with FM. Second, the multiple tests

Table 5 Combined allele frequencies and odds ratios in patients with fibromyalgia and healthy controls ${ }^{a}$

\begin{tabular}{|c|c|c|c|c|c|c|}
\hline Combined alleles & Controls, $n(\%)$ & Fibromyalgia, $n(\%)$ & Crude OR $(95 \% \mathrm{Cl})$ & $p$ value $^{\mathrm{b}}$ & Age and sex adjusted OR (95\% Cl) & $p$ value $^{\mathrm{b}}$ \\
\hline TGACCGCTGC & $193(26.6)$ & $340(45)$ & 1.0 (reference) & & 1.0 (reference) & \\
\hline TATCCAACCT & $119(16.4)$ & $232(30.7)$ & $1.107(0.834-1.469)$ & 0.483 & $1.106(0.833-1.47)$ & 0.487 \\
\hline TGACCACTGC & $160(22)$ & $1(0.1)$ & $0.004(0-0.026)$ & $<0.001$ & $0.004(0.0-0.026)$ & $<0.001$ \\
\hline TAACTACCCT & $66(9.1)$ & $146(19.3)$ & $1.256(0.894-1.765)$ & 0.19 & $1.248(0.887-1.756)$ & 0.204 \\
\hline TATCCGACCT & $103(14.2)$ & $0(0)$ & 0 (0-Inf) & 0.963 & 0 (0-Inf) & 0.963 \\
\hline TAACTGCCCT & $65(9)$ & $0(0)$ & $0(0-\ln f)$ & 0.971 & 0 (0-Inf) & 0.97 \\
\hline CAACCACCGC & $20(2.8)$ & $37(4.9)$ & $1.05(0.593-1.861)$ & 0.867 & $1.088(0.612-1.934)$ & 0.774 \\
\hline
\end{tabular}

Abbreviations: $O R$ odds ratio, $\mathrm{Cl}$ confidence interval

${ }^{a}$ Missing data were excluded $(n=51)$. Among 39 haplotype structures, 7 haplotypes with a frequency of at least $1 \%$ in both the patients and controls are presented; the total frequency of the other haplotype structures was $46(6 \%)$ for controls and $30(3.8 \%)$ for patients. Logistic regression models were used to calculate ORs

${ }^{\mathrm{b}}$ Computed for the estimated coefficient of each haplotype in the logistic regression 


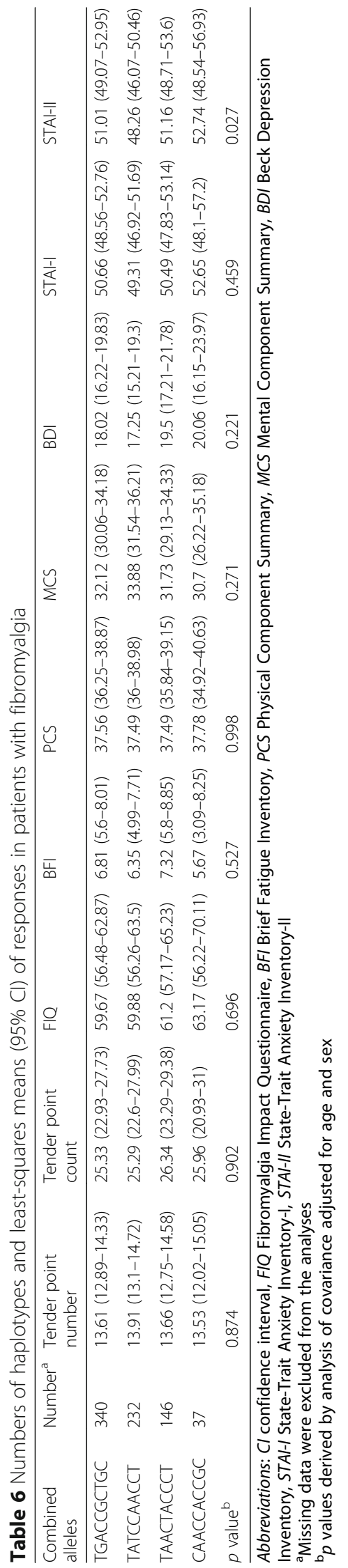


performed in this study may have increased the type I error. In genetics, controlling for multiple testing is important in estimating thresholds of significance accurately, particularly in genome-wide association studies (GWAS) [39]. However, in this target-gene-based SNP study, we did not consider the potential effects of multiple testing in the analyses. In fact, most published FM SNP case-control studies have not considered the potential effects of multiple testing. Third, we were unable to prospectively evaluate the associations between $B D N F$ genetic variation and clinical outcomes. Therefore, further studies are needed to investigate the effect of those genetic polymorphisms on the long-term clinical outcomes of patients with FM. Finally, to overcome the insufficient statistical power, we conducted a large-scale study involving $>800$ samples. However, our findings should be replicated in a larger population comprising multiple ethnicities.

\section{Conclusions}

In this study, we evaluated the association between $B D N F$ polymorphisms and FM in a large sample of the Korean population. We found that $B D N F$ gene polymorphisms influenced susceptibility to FM, and contributed to the severity of certain symptoms of FM. Further evidence from large prospective studies is needed to determine the generalizability of our findings to the broader population and their impact on the clinical outcomes of FM. Moreover, further work is needed to elucidate the biologic and epigenetic mechanisms underlying the complex role of the $B D N F$ gene in FM.

\section{Abbreviations \\ BDI: Beck Depression Inventory; BDNF: Brain-derived neurotrophic factor; BFI: Brief Fatigue Inventory; Cl: Confidence interval; DRG: Dorsal root ganglion; FIQ: Fibromyalgia Impact Questionnaire; FM: Fibromyalgia; ICF: Informed consent form; MCS: Mental Component Summary; NMDA: N- methyl-D-aspartate; NSAIDs: Nonsteroidal anti-inflammatory drugs; PCS: Physical Component Summary; SF-36: 36-Item Medical Outcomes Study Short-Form Health Survey; SNP: Single-nucleotide polymorphism; SNRI: Serotonin-norepinephrine reuptake inhibitors; SSRI: Selective serotonin reuptake inhibitor; STAI-I: State-Trait Anxiety Inventory-l; STAI-II: State-Trait Anxiety Inventory-II; TCA: Tricyclic antidepressant}

\section{Acknowledgements}

We would like to thank the patients and their families for participating in this study.

\section{Funding}

This study was supported by the Bio \& Medical Technology Development Program of the NRF funded by the Korean government, MSIP (2017M3A9E8023014), and by a grant (CRI16015-1) from Chonnam National University Hospital Biomedical Research Institute.

\section{Availability of data and materials}

The datasets used during the current study are available from the corresponding author on reasonable request.

\section{Authors' contributions}

D-J P and S-SL conceived and designed the study. S-HK, S-SN, JHL, S-KK, YAL, S-JH, H-SK, H-SL, HAK, C-IJ, and S-HK acquired data. D-J P and S-SL performed statistical analysis and drafted the manuscript. All authors critically revised the manuscript for important intellectual content. All authors read and approved the final manuscript.

\section{Ethics approval and consent to participate}

This study was conducted in accordance with the Declaration of Helsinki, and written informed consent was obtained from all participants at the time of recruitment. Exactly the same informed consult form (ICF) and study protocol were provided to the independent Institutional Review Board/Ethics Committee (IRB/EC) at each medical center, and each IRB/EC reviewed the appropriateness of the protocol and risks and benefits to the study participants. Ultimately, the IRB/EC at each medical center independently approved this study without revision of the ICF or study protocol.

\section{Consent for publication}

Not applicable.

\section{Competing interests}

The authors declare that they have no competing interests.

\section{Publisher's Note}

Springer Nature remains neutral with regard to jurisdictional claims in published maps and institutional affiliations.

\section{Author details}

'Division of Rheumatology, Department of Internal Medicine, Chonnam National University Medical School and Hospital, 42 Jebong-ro, Dong-gu, Gwangju 61469, Republic of Korea. ${ }^{2}$ Department of Internal Medicine, Inje University Haeundae Paik Hospital, Busan, Korea. ${ }^{3}$ Department of Internal Medicine, Soonchunhyang University, College of Medicine, Cheonan, Korea. ${ }^{4}$ Department of Internal Medicine, Maryknoll Medical Center, Busan, Korea. ${ }^{5}$ Department of Internal Medicine, Catholic University of Daegu, School of Medicine, Daegu, Korea. ${ }^{6}$ Department of Internal Medicine, School of Medicine, Kyung Hee University, Seoul, Korea. ${ }^{7}$ Department of Internal Medicine, Soonchunhyang University Seoul Hospital, Seoul, Korea. ${ }^{8}$ Hanyang University College of Medicine and the Hospital for Rheumatic Diseases, Seoul, Korea. ${ }^{9}$ Department of Allergy and Rheumatology, Ajou University Hospital, Ajou University School of Medicine, Suwon, Korea. ${ }^{10}$ Department of Internal Medicine, Konyang University Medical School, Daejeon, Korea.

${ }^{11}$ Departments of Internal Medicine, School of Medicine, Keimyung

University, Daegu, Korea.

Received: 27 March 2018 Accepted: 18 September 2018

Published online: 03 October 2018

\section{References}

1. Wolfe F, Smythe HA, Yunus MB, Bennett RM, Bombardier C, Goldenberg DL, et al. The American College of Rheumatology 1990 Criteria for the classification of fibromyalgia. report of the Multicenter Criteria Committee. Arthritis Rheum. 1990;33(2):160-72.

2. Jones GT, Atzeni F, Beasley M, Fluss E, Sarzi-Puttini P, Macfarlane GJ. The prevalence of fibromyalgia in the general population: a comparison of the American College of Rheumatology 1990, 2010, and modified 2010 classification criteria. Arthritis Rheumatol. 2015;67(2):568-75.

3. Maletic V, Raison CL. Neurobiology of depression, fibromyalgia and neuropathic pain. Front Biosci (Landmark Ed). 2009;14:5291-338.

4. Ablin JN, Buskila D. Update on the genetics of the fibromyalgia syndrome. Best Pract Res Clin Rheumatol. 2015;29(1):20-8.

5. Park DJ, Lee SS. New insights into the genetics of fibromyalgia. Korean J Intern Med. 2017;32(6):984-95.

6. Buskila D, Sarzi-Puttini P, Ablin JN. The genetics of fibromyalgia syndrome. Pharmacogenomics. 2007;8(1):67-74.

7. Park DJ, Kim SH, Nah SS, Lee JH, Kim SK, Lee YA, et al. Association between catechol-O-methyl transferase gene polymorphisms and fibromyalgia in a Korean population: a case-control study. Eur J Pain. 2016;20(7):1131-9.

8. Wu YJ, Kruttgen A, Moller JC, Shine D, Chan JR, Shooter EM, et al. Nerve growth factor, brain-derived neurotrophic factor, and neurotrophin-3 are sorted to dense-core vesicles and released via the regulated pathway in primary rat cortical neurons. J Neurosci Res. 2004;75(6):825-34.

9. Obata K, Noguchi K. BDNF in sensory neurons and chronic pain. Neurosci Res. 2006;55(1):1-10. 
10. Wu K, Len GW, McAuliffe G, Ma C, Tai JP, Xu F, et al. Brain-derived neurotrophic factor acutely enhances tyrosine phosphorylation of the AMPA receptor subunit GluR1 via NMDA receptor-dependent mechanisms. Brain Res Mol Brain Res. 2004;130(1-2):178-86.

11. Yajima Y, Narita M, Usui A, Kaneko C, Miyatake M, Yamaguchi T, et al. Direct evidence for the involvement of brain-derived neurotrophic factor in the development of a neuropathic pain-like state in mice. J Neurochem. 2005; 93(3):584-94.

12. Laske C, Stransky E, Eschweiler GW, Klein R, Wittorf A, Leyhe T, et al. Increased BDNF serum concentration in fibromyalgia with or without depression or antidepressants. J Psychiatr Res. 2007;41(7):600-5.

13. Zanette SA, Dussan-Sarria JA, Souza A, Deitos A, Torres ILS, Caumo W. Higher serum S100B and BDNF levels are correlated with a lower pressurepain threshold in fibromyalgia. Mol Pain. 2014;10:46.

14. Haas L, Portela LV, Bohmer AE, Oses JP, Lara DR. Increased plasma levels of brain derived neurotrophic factor (BDNF) in patients with fibromyalgia. Neurochem Res. 2010;35(5):830-4

15. Kim SK, Kim SH, Nah SS, Lee JH, Hong SJ, Kim HS, et al. Association of guanosine triphosphate cyclohydrolase 1 gene polymorphisms with fibromyalgia syndrome in a Korean population. J Rheumatol. 2013;40(3):316-22.

16. Okifuji A, Turk DC, Sinclair JD, Starz TW, Marcus DA. A standardized manual tender point survey. I. Development and determination of a threshold point for the identification of positive tender points in fibromyalgia syndrome. J Rheumatol. 1997;24(2):377-83.

17. Kim YA, Lee SS, Park K. Validation of a Korean version of the Fibromyalgia Impact Questionnaire. J Korean Med Sci. 2002;17(2):220-4.

18. Mendoza TR, Wang XS, Cleeland CS, Morrissey H, Johnson BA, Wendt JK, et al. The rapid assessment of fatigue severity in cancer patients - use of the brief fatigue inventory. Cancer. 1999;85(5):1186-96.

19. Richter P, Werner J, Heerlein A, Kraus A, Sauer H. On the validity of the Beck Depression Inventory. Rev Psychopathol. 1998;31(3):160-8.

20. Ware JE Jr, Sherbourne CD. The MOS 36-item short-form health survey (SF-36), I Conceptual framework and item selection. Med Care. 1992;30(6):473-83.

21. Kim JT, Shin DK. A study based on the standardization of the STAI for Korea. New Med J. 1978;21(11):69-75.

22. Malik-Hall M, Dina OA, Levine JD. Primary afferent nociceptor mechanisms mediating NGF-induced mechanical hyperalgesia. Eur J Neurosci. 2005; 21(12):3387-94.

23. Kerr BJ, Bradbury EJ, Bennett DLH, Trivedi PM, Dassan P, French J, et al. Brainderived neurotrophic factor modulates nociceptive sensory inputs and NMDAevoked responses in the rat spinal cord. J Neurosci. 1999;19(12):5138-48.

24. Siniscalco D, Giordano C, Rossi F, Maione S, de Novellis V. Role of neurotrophins in neuropathic pain. Curr Neuropharmacol. 2011;9(4):523-9.

25. Karege F, Perret G, Bondolfi G, Schwald M, Bertschy G, Aubry JM. Decreased serum brain-derived neurotrophic factor levels in major depressed patients. Psychiatry Res. 2002;109(2):143-8.

26. Yoshimura R, Mitoma M, Sugita A, Hori H, Okamoto T, Umene W, et al. Effects of paroxetine or milnacipran on serum brain-derived neurotrophic factor in depressed patients. Prog Neuro-Psychopharmacol Biol Psychiatry. 2007;31(5):1034-7.

27. Sarchielli P, Mancini ML, Floridi A, Coppola F, Rossi C, Nardi K, et al. Increased levels of neurotrophins are not specific for chronic migraine: evidence from primary fibromyalgia syndrome. J Pain. 2007;8(9):737-45.

28. Caumo W, Deitos A, Carvalho S, Leite J, Carvalho F, Dussan-Sarria JA, et al. Motor cortex excitability and BDNF levels in chronic musculoskeletal pain according to structural pathology. Front Hum Neurosci. 2016;10:357.

29. Fallon N, Chiu Y, Nurmikko T, Stancak A. Functional connectivity with the default mode network is altered in fibromyalgia patients. PLoS One. 2016; 11(7):e0159198.

30. Hsiao FJ, Wang SJ, Lin YY, Fuh JL, Ko YC, Wang PN, et al. Altered insuladefault mode network connectivity in fibromyalgia: a resting-state magnetoencephalographic study. J Headache Pain. 2017;18(1):89.

31. Jang JH, Yun JY, Jung WH, Shim G, Byun MS, Hwang JY, et al. The impact of genetic variation in comt and bdnf on resting-state functional connectivity. Int J Imaging Syst Technol. 2012;22(1):97-102.

32. Xiao Y, Russell IJ, Liu YG. A brain-derived neurotrophic factor polymorphism Val66Met identifies fibromyalgia syndrome subgroup with higher body mass index and C-reactive protein. Rheumatol Int. 2012;32(8):2479-85.

33. International HapMap C. A haplotype map of the human genome. Nature. 2005:437(7063):1299-320
34. Diatchenko L, Nackley AG, Slade GD, Bhalang K, Belfer I, Max MB, et al. Catechol-O-methyltransferase gene polymorphisms are associated with multiple pain-evoking stimuli. Pain. 2006;125(3):216-24.

35. Martinez-Jauand M, Sitges C, Rodriguez V, Picornell A, Ramon M, Buskila D, et al. Pain sensitivity in fibromyalgia is associated with catechol-Omethyltransferase (COMT) gene. Eur J Pain. 2013;17(1):16-27.

36. Vargas-Alarcon G, Fragoso JM, Cruz-Robles D, Vargas A, Lao-Villadoniga Jl, GarciaFructuoso F, et al. Catechol-O-methyltransferase gene haplotypes in Mexican and Spanish patients with fibromyalgia. Arthritis Res Ther. 2007;9(5):R110.

37. Park DJ, Kim SH, Nah SS, Lee JH, Kim SK, Lee YA, et al. Polymorphisms of the TRPV2 and TRPV3 genes associated with fibromyalgia in a Korean population. Rheumatology (Oxford). 2016;55(8):1518-27.

38. Arnold LM, Hudson II, Keck PE, Auchenbach MB, Javaras KN, Hess EV. Comorbidity of fibromyalgia and psychiatric disorders. J Clin Psychiatry. 2006:67(8):1219-25.

39. Clarke GM, Anderson CA, Pettersson FH, Cardon LR, Morris AP, Zondervan KT. Basic statistical analysis in genetic case-control studies. Nat Protoc. 2011; 6(2):121-33.
Ready to submit your research? Choose BMC and benefit from:

- fast, convenient online submission

- thorough peer review by experienced researchers in your field

- rapid publication on acceptance

- support for research data, including large and complex data types

- gold Open Access which fosters wider collaboration and increased citations

- maximum visibility for your research: over $100 \mathrm{M}$ website views per year

At BMC, research is always in progress.

Learn more biomedcentral.com/submissions 